\title{
Stratosphere-troposphere exchange: A zonal mean perspective of angular momentum
}

\author{
Joseph Egger $^{\mathrm{a}, *}$, Klaus-Peter Hoinka ${ }^{\mathrm{b}}$ \\ a Meteorologisches Institut der Universität München, Theresienstrs. 37, 80333 München, Germany \\ b Institut für Physik der Atmosphäre, DLR, Oberpfaffenhofen, Germany
}

Available online 7 February 2007

\begin{abstract}
The exchange of axial angular momentum (AM) between stratosphere and troposphere forms an important part of stratosphere-troposphere exchange (STE). Data are used to quantify the related fluxes through an idealized tropopause for specific flux events both for the global tropopause and regional segments. Time mean fluxes are considered as well. These are largest in the tropics and closely tied to mass fluxes. The data accuracy is found to be insufficient for a closure of the momentum budget of the Brewer-Dobson circulation. Positive regional flux events at the tropical tropopause are partly due to a short lived intensification of the Hadley circulation, but are also embedded in a rather long lived circulation anomaly which leads to a gain of positive AM in the lower tropical stratosphere. Moreover, there are large fluxes through the 'tropopause breaks'. Flux events at midlatitude segments involve also strong horizontal fluxes through the 'tropopause breaks', which override an otherwise downward flux from the stratosphere. These events are closely linked to tropical anomalies of AM, so that all 'regional' events turn out to be of global character. Data accuracy becomes a problem when fluxes through a global tropopause are investigated where substantial uncertainties are encountered near the 'tropopause breaks'.

All cases discussed exhibit a distinct similarity with deep anomalies of AM in the tropics sandwiched between anomalous AM columns of opposite sign to the north and south. There is always a center of flux convergence or divergence in the lower stratosphere. This suggests that all the events of large scale-angular momentum exchange between stratosphere and troposphere as analysed here follow a distinct dynamic pattern, which appears not to have been described before.
\end{abstract}

(C) 2007 Elsevier B.V. All rights reserved.

Keywords: Angular momentum; Stratosphere-troposphere exchange; Tropopause; Tropics

* Corresponding author. Tel.: +49 89 4570; fax: +49 892805508.

E-mail address: J.Egger@1rz-uni-muenchen.de (J. Egger). 


\section{Introduction}

In the past, the exchange of air and trace substances between stratosphere and troposphere has been investigated intensively (see Holton et al., 1995 and Haynes, 2005 for reviews). Much of the chemistry for stratospheric ozone depletion, for example, is induced by anthropogenic trace gases originating in the troposphere. Tropospheric ozone concentrations are affected by ozone transports down from the stratosphere. Estimates of the exchange rates are generally obtained with respect to a control surface in the lower stratosphere. For example, the $100 \mathrm{hPa}$ surface was selected by Rosenlof and Holton (1993) for an evaluation of the mass transfer. In principle, the tropopause is the appropriate control surface separating stratosphere and troposphere. Flux calculations across such a dynamically active surface are notoriously difficult. The calculation of trajectories and corresponding mass fluxes appears to be the most accurate method at the moment (Wirth and Egger, 1999; Wernli and Bourqui, 2002; Stohl et al., 2003; Fueglistaler et al., 2004). James et al. (2003) include even transports by subgrid-scale convection and turbulence.

Given this interest in stratosphere-troposphere exchange (STE), it is surprising that exchange rates of angular momentum (AM) have not been evaluated as yet. This exchange of angular momentum is of obvious importance both for the stratosphere and the troposphere. For example, the quasibiennial oscillation is driven by tropospheric waves transporting momentum into the stratosphere (e.g., Baldwin et al., 2001). Stratospheric warmings involve vertical AM fluxes. Orographically induced gravity waves transport momentum across the tropopause, which is deposited higher up during wave breaking events.

This neglect of AM exchange may be due to the fact that axial angular momentum

$$
\mu=\rho(u+\Omega a \cos \varphi) a \cos \varphi
$$

written in standard notation, is not a trace substance. Angular momentum is not materially conserved and flux estimates via trajectory calculations are not feasible. In particular, AM can be transported by waves. However, angular momentum is conserved in the sense that the total angular momentum of the stratosphere above a control surface can change only via fluxes through this surface. More specifically, the axial AM equation

$$
\frac{\partial}{\partial t} \mu+\nabla_{3} \cdot(\boldsymbol{v} \mu)=-\frac{\partial p}{\partial \lambda}
$$

$(\boldsymbol{v}$, wind velocity; $p$, pressure; $\lambda$, longitude) yields the total AM budget

$$
\frac{\mathrm{d}}{\mathrm{d} t} \int_{V} \mu \mathrm{d} v=\int_{S} \mu\left(\boldsymbol{v}_{\mathrm{S}}-\boldsymbol{v}\right) \cdot \boldsymbol{n} \mathrm{d} S-\int_{S} \frac{\partial h_{\mathrm{S}}}{\partial \lambda} p_{\mathrm{S}} \mathrm{d} S
$$

of the volume $V$ above the control surface $S$ which may, in particular, represent the tropopause $\left(v_{\mathrm{S}}\right.$ velocity of the control surface; $\boldsymbol{n}$ unit vector normal to $S$ pointing downward; $h_{\mathrm{S}}$ height of $S$; $p_{\mathrm{S}}$ pressure at $S$ ). The flux of angular momentum through $S$ is given by the first integral on the right hand side. The second integral is the torque exerted at the control surface. No upper control surface is included in (1.3) for the sake of simplicity. In what follows we assume that $h_{\mathrm{S}}$ does not depend on longitude and $\mathrm{S}$ does not move, that is, $\boldsymbol{v}_{\mathrm{S}}=0$. If, in particular, $h_{\mathrm{S}}$ does not vary with latitude (1.3) gives

$$
\frac{\mathrm{d}}{\mathrm{d} t} \int_{v} \mu \mathrm{d} v=\int_{S_{\mathrm{h}}} \mu \mathrm{wdS}
$$


Table 1

Control surfaces as selected

\begin{tabular}{llll}
\hline Control surface: & $\varphi_{1}$ & $\varphi_{2}$ & Height \\
\hline$S_{\mathrm{h}}$ & $90^{\circ} \mathrm{S}$ & $90^{\circ} \mathrm{N}$ & $h$ \\
$S_{\mathrm{c}}$ & $90^{\circ} \mathrm{S}$ & $90^{\circ} \mathrm{N}$ & $10 \mathrm{~km}$ for $|\varphi|>27^{\circ} ; 16 \mathrm{~km}$ for $|\varphi| \leq 27^{\circ}$ \\
$\Sigma_{\mathrm{T}}$ & $18^{\circ} \mathrm{S}$ & $18^{\circ} \mathrm{N}$ & $16 \mathrm{~km}$ \\
$\Sigma_{\mathrm{NH}}$ & $27^{\circ} \mathrm{N}$ & $63^{\circ} \mathrm{N}$ & $10 \mathrm{~km}$ \\
$\Sigma_{\mathrm{SH}}$ & $63^{\circ} \mathrm{S}$ & $27^{\circ} \mathrm{N}$ & $10 \mathrm{~km}$ \\
\hline
\end{tabular}

with vertical velocity $w$. The symbol $S_{h}$, where $h$ is given in kilometres, denotes this type of control surface. In addition, a climatological mean position $S_{\mathrm{c}}$ of the global tropopause will be prescribed in some cases with $h_{\mathrm{S}}=10 \mathrm{~km}$ for $|\varphi| \geq 27^{\circ}$ and $h_{\mathrm{S}}=16 \mathrm{~km}$ for $|\varphi|<27^{\circ}$ (see, for example, Fig. 4). There are 'tropopause breaks' at $|\varphi|=27^{\circ}$. In that case, the horizontal flux contributions

$$
F_{1,2}=\int_{10}^{15} \mu v \mathrm{~d} z
$$

at $\varphi= \pm 27^{\circ}$ must be added to the vertical fluxes at $z=h_{\mathrm{S}}$ where $v$ is the meridional velocity. Of course, even this refined meridional profile $S_{\mathrm{c}}$ of the control surface is at best a crude approximation to the structure of the extra-tropical tropopause and the tropical tropopause layer (Highwood and Hoskins, 1998). In other words, we accept the atmospheric domain above $S$ as an approximate stratosphere, the angular momentum of which we want to study. Unlike the global angular momentum balance, that of this stratosphere is free of torques acting at the surface. Instead, we have fluxes through $S$. All the control surfaces $S$ considered in this paper are listed in Table 1 .

In principle, an evaluation of the AM flux through a properly defined realistic tropopause is possible although the computational expense would be considerable. On the other hand, it is doubtful if the data quality is sufficiently high to warrant this effort. As will be seen, the accuracy of the transports available from the analyses is rather limited.

It is a useful convention to split $\mu$ in a wind term

$$
\mu_{\mathrm{w}}=\rho u a \cos \varphi
$$

representing the relative angular momentum and a mass term

$$
\mu_{\mathrm{m}}=\rho \Omega a^{2} \cos ^{2} \varphi
$$

also called $\Omega$-term, representing the angular momentum of the daily rotation. Of course (1.1), (1.4) and (1.7) imply that any mass exchange involves also exchanges of angular momentum. One simply has to multiply the zonal mean vertical mass flux by the geometric factor $\Omega a^{2} \cos ^{2} \varphi$ to arrive at an angular momentum flux. This procedure neglects wind term transports, of course. Here, we evaluate the total transport of angular momentum for climatic mean conditions and for episodes.

From a theoretical point of view, Haynes et al. (1991) addressed the angular momentum exchange between troposphere and stratosphere within the framework of the 'downward control' principle (see also Shepherd and Shaw, 2004; Haynes, 2005). Averaging (1.2) over longitude and 
time yields in steady state

$$
\nabla_{3} \cdot(\hat{\boldsymbol{v}} \hat{\mu})=-\nabla_{3} \cdot\left(\hat{\boldsymbol{v}}^{\prime} \mu^{\prime}\right)
$$

where the symbol ^denotes the mean (' deviation). The divergence of the angular momentum flux due to the mean motion must balance that of the eddy fluxes, that is, the 'eddy forcing' on the right hand side of (1.8). Haynes et al. (1991) demonstrated on the basis of (1.8), that the mean vertical motion at the tropopause can be calculated provided the 'wave forcing' in the stratosphere is known.

While the climatological mean state is certainly of interest short-term episodes are important as well. This has been realized since long in work on STE. Great efforts have been made to quantify the mass exchange during tropopause folds (e.g., Lamarque and Hess, 1994; Traub and Lelieveld, 2003), cut-off lows (Price and Vaughan, 1993) and other synoptic and mesoscale processes. Climatologies of such events and the related mass exchanges are emerging (Sprenger et al., 2005).

\section{Methods and data}

The zonal mean of (1.2) is

$$
\frac{\partial}{\partial t} \bar{\mu}+a^{-1} \frac{\partial}{\partial \varphi} V+\frac{\partial}{\partial z} W=0
$$

where

$$
\bar{\mu}=\int_{0}^{2 \pi} \mu a \cos \varphi \mathrm{d} \lambda
$$

and

$$
\begin{aligned}
& V=\overline{v \mu}=V_{\mathrm{m}}+V_{\mathrm{w}} \\
& W=\overline{w \mu}=W_{\mathrm{m}}+W_{\mathrm{w}}
\end{aligned}
$$

are the mean fluxes which may be split in a flux of the mass term and of the wind term. Note that the zonal integral of the pressure gradient term in (1.2) does not necessarily vanish because the $z$-surfaces intersect the mountains. The related mountain torque is given below. With that (1.4) becomes

$$
\frac{\mathrm{d}}{\mathrm{d} t} M_{\mathrm{S}}=\left.\int \mathrm{Wad} \varphi\right|_{h=h_{\mathrm{S}}}
$$

where

$$
M_{\mathrm{S}}=\int_{-\pi / 2}^{\pi / 2} \int_{h_{S}}^{\infty} \bar{\mu} a \mathrm{~d} \varphi \mathrm{d} z
$$

is the angular momentum of the 'stratosphere' and where the torque on the stratosphere is omitted. The corresponding tropospheric angular momentum $M_{\mathrm{T}}$ is affected by friction and mountain 
torques $T_{\mathrm{f}}$ and $T_{\mathrm{m}}$ so that

$$
\frac{\mathrm{d}}{\mathrm{d} t} M_{\mathrm{T}}=-\int_{-\pi / 2}^{\pi / 2} \mathrm{~W} a \mathrm{~d} \varphi+T_{\mathrm{f}}+T_{\mathrm{m}}
$$

where

$$
T_{\mathrm{f}}=\int_{A} \tau_{\lambda} a \cos \varphi \mathrm{d} A
$$

( $\tau_{\lambda}$ zonal surface stress; $A$ surface of the earth) and

$$
T_{\mathrm{m}}=\int_{A} h \frac{\partial p_{\mathrm{B}}}{\partial \lambda} \mathrm{d} A
$$

with surface pressure $p_{\mathrm{B}}$ and topography $h$.

Peixoto and Oort (1992) evaluated the seasonal mean fluxes of angular momentum as a function of latitude and height. Vertical transports were calculated as residuals. Here, transport data are directly available. In particular, the choice of the $z$-system gives immediate access to vertical fluxes of the mass term.

It is customary to define events via synoptic processes. For example, Sprenger et al. (2005) look at the STE related to cut-off lows and streamers. These synoptic features are identified on maps by a search algorithm and the related fluxes are evaluated. Here, we choose a different approach by defining a flux event and evaluating the flow features related to it. This strategy is partly enforced by the fact that we have only zonally averaged fields at our disposal. Cut-off lows cannot be identified within this framework. On the other hand, we are free from the somewhat arbitrary choice of synoptic events. Events of vertical fluxes through a section $\Sigma$ of $S$ are singled out by introducing the angular momentum flux

$$
F\left(\varphi_{1}, \varphi_{2}\right)=\int_{\Sigma} \boldsymbol{V} \cdot n \mathrm{~d} \Sigma
$$

through the control surface $\Sigma$ as a parameter. The control-surfaces as chosen in this paper are listed in Table 1. In particular, global cases are considered as well. The flux $F$ is normalized by its standard deviation $\sigma_{F}$. Let us define by $\mathrm{C}(b, c \mid \tau)$ the covariance function of $a$ variable $b$ with variable $c$ where $b$ leads with lag $\tau$. Events of strong fluxes are captured in a statistical sense via the autocorrelation function. The flux peaks at $\tau=0$ and decays with increasing lag (see Fig. 2). A typical length of an event can be derived from the decay time of the autocorrelation. Moreover, we can distinguish between positive flux events with $F>0$ and negative ones by introducing the fluxes

$$
F^{+}=(F+|F|), \quad F^{-}=(F-|F|),
$$

where the factor $1 / 2$ is omitted so that $F^{+}$and $F^{-}$have about the same variance as $F$.

The flow and transport patterns related to $F$ are obtained by looking at the covariance equations relating $\bar{\mu}, V$ and $W$ to $F$. Multiplication of (2.1) by $F(t-\tau)$, redefinition of time and forming expectations gives

$$
\frac{\partial}{\partial \tau} C(F, \bar{\mu} \mid \tau)+a^{-1} \frac{\partial}{\partial \varphi} C(F, V \mid \tau)+\frac{\partial}{\partial z} C(F, W \mid \tau)=0 .
$$


Corresponding equations are valid for $F^{+}$and $F^{-}$. All these equations will be analysed on the basis of the ERA-15 data set covering the period January 1979-December 1993 generated by the European Center for Medium-Range Weather Forecasts Reanalysis Project (Gibson et al., 1997). Wind and density are available at the 31 levels of the hybrid $\sigma$-system of the ERA-scheme. Torques at the earth's surface can be calculated on the basis of these data. The data to be used in the calculations are averages over the four values per day originally available. All data are transformed to equidistant $z$-surfaces with a vertical spacing of $D z=1000 \mathrm{~m}$. This way, the mass terms become available throughout the atmosphere while they reduce to a surface integral in pressure and/or sigma coordinates. The original data are available with a horizontal resolution of $1.125^{\circ} \times 1.125^{\circ}$. We remove small-scale horizontal variability of the fluxes by introducing latitude belts of width $a D \varphi=1000 \mathrm{~km}$. The fluxes $V, W$ are averaged such that large-scale budgets of angular momentum can be evaluated for grid boxes of area $a D \varphi \times D z$ with indices $(i, j)$ where

$$
-\frac{\pi}{2}+(i-1) D \varphi \leq \varphi \leq-\frac{\pi}{2}+i D \varphi
$$

covers the width and

$$
(j-1) D z<z<j D z
$$

the depth of a box $(1 \leq i \leq 20 ; 1 \leq j \leq 28)$. If, in particular (2.12), is integrated over the area of a box it follows

$$
\frac{\mathrm{d}}{\mathrm{d} \tau} C\left(F, \tilde{\mu}_{i j} \mid \tau\right)+C\left(F,\left(\tilde{V}_{i+1, j}-\tilde{V}_{i, j}+\tilde{W}_{i, j+1}-W_{i, j}\right) \mid \tau\right)=0
$$

where $\tilde{\mu}_{i j}$ is the integral of $\bar{\mu}$ over the area of the box $(I, j)$ and $\tilde{V}_{i+1, j}\left(\tilde{W}_{i, j+1}\right)$ is the integral of $V(W)$ over its northern (upper) boundary). With (2.15) we concentrate on the large-scale aspects of the angular momentum budget. Note, in particular, that (2.1) does not contain a term representing vertical momentum fluxes $\hat{v^{\prime}} \mu^{\prime}$ due to gravity waves as in (1.8). Such information is partly contained in the gravity wave drag data of the ERA set, however, which does not provide information on the level of wave breaking. In other words, the data needed to check (1.8) are not available.

Note that the fluxes $V$ and $W$ contain all information stored in the original data except for interpolation errors. Even so, the ERA-15 data are, of course, strongly model dependent and the deviation of the analysed fluxes from the real ones is not known. This choice of the analysis grid in space and time ensures that we look here essentially at relatively slow events of STE. James et al. (2003) found that about $90 \%$ of all cross-tropopause flows return within $6 \mathrm{~h}$. Such fluxes are automatically excluded here.It is useful (see Egger and Hoinka, 2005b) to introduce a generalized streamfunction $\psi$ and velocity potential $\chi$ per box $(i, j)$ via

$$
\begin{aligned}
& C\left(F, \tilde{V}_{i, j} \mid \tau\right)=\psi_{i, j}-\psi_{i, j+1}+\chi_{i, j}-\chi_{i-1, j}, \\
& C\left(F, \tilde{W}_{i, j} \mid \tau\right)=\psi_{i+1, j}-\psi_{i, j}+\chi_{i, j}-\chi_{i, j-1} .
\end{aligned}
$$

Given observed covariance functions at the left it is straightforward to determine $\psi_{i, j}$ and $\chi_{i, j}$ where the streamfunction represents that part of the angular momentum fluxes which has no impact on changes of the angular momentum. The velocity potential describes that part of fluxes which affects $\mu$. At the ground $(j=1)$ we have

$$
C\left(F_{\mathrm{F}}, \tilde{W}_{i, 1} \mid \tau\right)=C\left(F_{\mathrm{F}}, T_{i} \mid \tau\right)
$$


where $T_{i}=T_{\mathrm{fi}}+T_{\mathrm{mi}}$ is the sum of the friction and mountain torque per belt. These torques are not included in (2.15) because this equation is strictly valid only above the top of the earth's mountains. A more complete version of this equation is given in Egger and Hoinka (2005b), where the boundary conditions for $\psi$ and $\chi$ are discussed in detail. Note, in particular that $\chi$ is evaluated directly from the tendencies via

$$
\frac{\mathrm{d}}{\mathrm{d} \tau} C\left(F, \tilde{\mu}_{i j} \mid \tau\right)+\chi_{i+1, j}+\chi_{i-1, j}+\chi_{i, j+1}+\chi_{i, j-1}-4 \chi_{i j}=0
$$

because these tendencies appear to be more reliable than the flux divergences in (2.15). The total flux vector is then composed of a $\psi$-flux parallel to the "streamlines" $\psi=$ const. and a $\chi$-flux normal to the isolines of $\chi$.

The strategy proposed here is similar to that where composites are formed with respect to extreme values of a parameter. For example, Kim and Lee (2004) select events with high momentum flux convergence at a certain height and latitude to study baroclinic wave development in the southern hemisphere. But unlike Kim and Lee (2004) we allow all values of $F$ to enter the analysis, so that the statistical covariance equation (2.15) can be used. Also by considering $F^{+}$ and $F^{-}$, we are able to distinguish between positive and negative flux events just as is possible in the composite technique.

After discussing the time mean situation (Section 3.1), we will look at flux events with respect to certain segments of the tropopause (Section 3.2 and 3.3) and finally at events involving the global tropopause (Section 3.4).

\section{Results}

\subsection{Climatology}

The basic features of the vertical annual mean fluxes $W_{\mathrm{w}}$ and $W_{\mathrm{m}}$ of the wind and mass terms are given in Table 2. The wind term fluxes are of the order of a few Hadley (1 Hadley $=10^{18} \mathrm{~J}$ ) while those of the mass term can be as large as 300 Hadley. A feeling for the relative importance of these fluxes can be obtained by recalling that a flux of 1 Hadley has to continue for $\sim 10$ days to increase the flow speed factor $u_{\mathrm{o}}$ of a superrotating stratosphere with $u=u_{\mathrm{o}} \cos \varphi$ by $\sim 1 \mathrm{~ms}^{-1}$. This estimate assumes that the flux affects only the wind term but not the mass term.

There is a moderate extratropical positive wind term flux $W_{\mathrm{w}}$ in both hemispheres. This flux is negative in the tropical belt $|\varphi| \leq 27^{\circ}$ where easterly momentum ascends. The wind term fluxes are dwarfed by those of the mass term. There are particularly large positive mass term fluxes close to the equator with negative branches to the north and south. These fluxes are, of course, tied to the Hadley circulation. The total mass flux term in the tropical belt is positive as expected and amounts to $\sim 140$ Hadley at $z=9 \mathrm{~km}$ while that of the wind term is $\sim-14$ Hadley with a ratio of both mean fluxes $R \sim-10$. The guess $R \sim \Omega a / \bar{u}$, where $\bar{u}$ is a mean tropical flow velocity would give excessively large easterly winds. In other words, eddy momentum fluxes contribute to the total wind term flux. In general, there is a decrease of flux intensity with height, which reflects inter alia the corresponding decrease of density but also the weakening of the meridional circulation. The total equatorial mass term flux amounts to 22 Hadley at $z=15 \mathrm{~km}$, that is, near the tropical tropopause.

The last column in Table 2 contains the global sum which represents, of course, the balance (1.8) except that the fluxes due to unresolved motions are not represented. Nevertheless, the residual is quite small both for the mass and the wind term. This is a surprising result for two reasons. First, 
Table 2

Mean vertical fluxes of wind term/mass term in units of Hadley $\left(=10^{18} \mathrm{~J}\right)$ at the heights $h_{\mathrm{S}}$ indicated in the first column in belts of $1000 \mathrm{~km}$ width. The latitudes on top give the northern boundary of a belt

\begin{tabular}{|c|c|c|c|c|c|c|c|c|c|c|c|c|c|c|c|c|c|}
\hline$h_{\mathrm{S}}(\mathrm{km})$ & $63^{\circ} \mathrm{S}$ & $54^{\circ} \mathrm{S}$ & $45^{\circ} \mathrm{S}$ & $36^{\circ} \mathrm{S}$ & $27^{\circ} \mathrm{S}$ & $18^{\circ} \mathrm{S}$ & $9^{\circ} \mathrm{S}$ & $0^{\circ}$ & $9^{\circ} \mathrm{N}$ & $18^{\circ} \mathrm{N}$ & $27^{\circ} \mathrm{N}$ & $36^{\circ} \mathrm{N}$ & $45^{\circ} \mathrm{N}$ & $54^{\circ} \mathrm{N}$ & $63^{\circ} \mathrm{N}$ & $72^{\circ} \mathrm{N}$ & Sum \\
\hline 17 & $0 / 1$ & $0 / 0$ & $0 /-4$ & $-0 /-4$ & $0 /-2$ & $0 / 3$ & $0 / 5$ & $0 / 4$ & $0 / 2$ & $0 / 6$ & $0 / 4$ & $0 /-2$ & $0 /-4$ & $-0 /-3$ & $0 /-1$ & $0 / 1$ & $0 / 1$ \\
\hline 15 & $0 / 1$ & $0 / 0$ & $0 /-4$ & $-1 /-7$ & $-1 /-8$ & $-1 /-4$ & $0 / 7$ & $0 / 9$ & $0 / 14$ & $0 / 25$ & $-1 /-2$ & $-1 /-8$ & $-1 /-6$ & $0 /-3$ & $0 / 0$ & $0 / 0$ & $-1 / 1$ \\
\hline 13 & $0 / 1$ & $0 / 1$ & $0 /-3$ & $0 /-8$ & $-2 /-22$ & $-3 /-40$ & $-1 /-6$ & $-1 / 38$ & $-1 / 84$ & $-1 / 52$ & $-3 /-38$ & $-2 /-28$ & $0 /-6$ & $0 /-2$ & $0 / 1$ & $0 / 0$ & $-1 / 3$ \\
\hline 11 & $0 / 2$ & $1 / 3$ & $1 / 2$ & $2 /-8$ & $0 /-45$ & $-5 /-106$ & $-3 /-35$ & $-2 / 87$ & $-2 / 217$ & $-3 / 56$ & $-5 /-99$ & $-3 /-51$ & $1 /-5$ & $1 / 2$ & $4 / 3$ & $2 / 1$ & $-1 / 3$ \\
\hline 9 & $0 / 3$ & $1 / 9$ & $3 / 10$ & $4 /-16$ & $3 /-67$ & $-3 /-139$ & $-2 /-48$ & $-1 / 114$ & $-2 / 270$ & $-2 / 61$ & $-4 /-121$ & $-1 /-65$ & $2 /-7$ & $1 / 6$ & $1 / 8$ & $0 / 2$ & $0 / 2$ \\
\hline
\end{tabular}




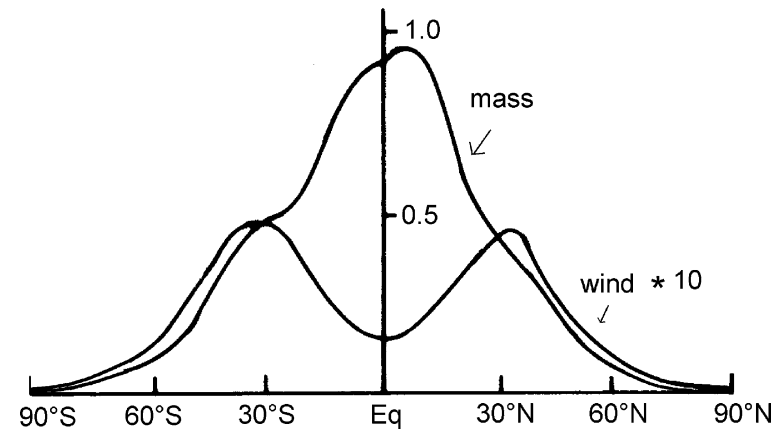

Fig. 1. Standard deviations of the vertical mass and wind term fluxes through belts of $1000 \mathrm{~km}$ width at a height of $10 \mathrm{~km}$ in $10^{2}$ Hadley; note that the wind term fluxes are multiplied by a factor of 10 .

the mean gravity wave drag is -5.8 Hadley (Egger and Hoinka, 2002) in the ERA-15 set. It is likely that most of this flux reaches the stratosphere, so that there is a sink of $\sim 5.6$ Hadley which is not balanced by the resolved fluxes.

Second, the conservation of the stratospheric mass term $M_{\mathrm{sm}}$ as implied by Table 2 is not compatible with the Brewer-Dobson circulation. After all, the mass term varies in time according to

$$
\frac{\mathrm{d} M_{\mathrm{sm}}}{\mathrm{d} t}=-\int_{v} 2 \Omega a \sin \varphi \cos \varphi \rho v \mathrm{~d} V
$$

so that the poleward directed Brewer-Dobson circulation implies a permanent loss of the stratospheric mass term and a gain of the wind term. An evaluation of the conversion term in (3.1) gives -9.7 Hadley for $h_{\mathrm{S}}=9 \mathrm{~km}\left(-5.6\right.$ Hadley for $\left.h_{\mathrm{S}}=17 \mathrm{~km}\right)$. This loss of the mass term is not balanced by corresponding imports in Table 2. Vice versa (3.1) implies a permanent gain for the wind terms, which must be balanced by exports. There is no possibility to resolve this issue with the available data.

The standard deviation of the vertical fluxes is displayed in Fig. 1 for $1000 \mathrm{~km}$-belts at $\mathrm{S}_{10}$. Those of the mass term are about one order of magnitude larger than those of the wind term. The variability of the mass term transports is largest at the equator that of the wind term fluxes peaks in the subtropics. The standard deviation of the fluxes through the two vertical sections of $S_{\mathrm{c}}$ is 46 Hadley.

The annual cycle of the transports is, of course, also dominated by that of the mass term and it is the Hadley circulation where fluxes are greatest by far (see Egger and Hoinka, 2005a and references therein).

\subsection{Tropical flux events}

Angular momentum flux events in the tropics are studied by choosing a segment $\Sigma_{\mathrm{T}}$ of $S_{\mathrm{c}}$ near the equator as control surface (Table 1; see also Figs. 4-6). These events are fairly longlived as can be seen from the autocorrelation of the flux in Fig. 2. There is a rapid decrease within the first two days, but the following decay is rather slow. The standard deviation is 12 Hadley so that substantial fluxes occur. Of course, the resulting impact on the stratospheric angular momentum $M_{\mathrm{S}}$ depends on the choice of the stratospheric domain. If $S_{16}$ is chosen as the lower boundary, the 'stratosphere' coincides roughly with the over-world as introduced by Hoskins (1991) to denote those parts of the 


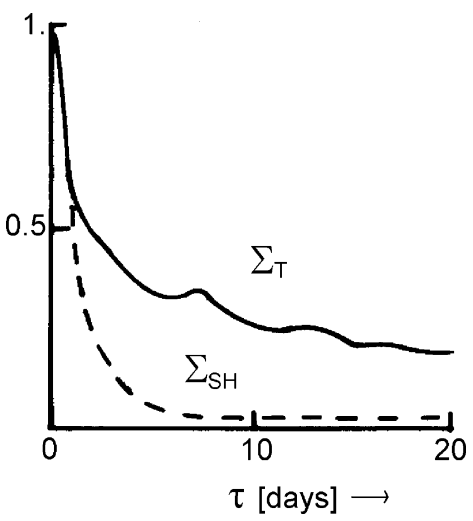

Fig. 2. Autocorrelation of the angular momentum flux through $\Sigma_{\mathrm{T}}\left(\sigma_{F}=11\right.$ Hadley $)$ and $\Sigma_{\mathrm{SH}}\left(\sigma_{\mathrm{F}}=40\right.$ Hadley $)$ for lags $\tau \leq 20$ days.

stratosphere which are not connected to the ground via isentropic surfaces. In that case we observe a monotonic and simultaneous increase of both components of the stratospheric global angular momentum (Fig. 3 ) from $\tau=-10$ days till $\tau=10$ days. This implies that the 'conversion term' in (3.1) is small as may be expected for a tropical event. There is a total increase of $M_{\mathrm{S}}$ by $6 \times 10^{5}$ Hadley s within the interval $-10 \leq \tau \leq 10$ days, while a typical flux event provides an input of $\sim 4 \times 10^{5}$ Hadley s as follows from an integration of the autocorrelation in Fig. 2. This discrepancy does not pose a problem because the flux through $\Sigma_{\mathrm{T}}$ is not the only one across $S_{16}$. Here and in what follows we assume in our discussion that fluxes are positive, if not stated otherwise.

A somewhat different picture emerges for $S=S_{\mathrm{c}}$ (Fig. 3). The stratospheric mass term is at a fairly high level at $\tau=-10$ days and grows weakly till $\tau \sim-2$ days. Obviously, mass is stored in the extratropical part of the layers $10 \leq z \leq 16 \mathrm{~km}$ which are excluded from the analysis for $\mathrm{S}=S_{16}$. There is a short decrease till $\tau=2$ days followed by further increase. The sharp peak of the flux through $\Sigma_{\mathrm{T}}$ at $\tau=0$ (Fig. 2) is not reflected in a corresponding increase of $M_{\mathrm{S}}$. This implies that there exists concomitant flux back to the troposphere through the vertical parts of the tropopause (see also Fig. 6). The mass terms for $S_{\mathrm{c}}$ and $S_{16}$ coincide almost for $\tau \geq 2$ days. The wind term behaves quite similar to that for $S_{16}$. The total increase of stratospheric angular momentum is $5 \times 10^{5}$ Hadley s. This rather small discrepancy with respect to the results for $S_{16}$

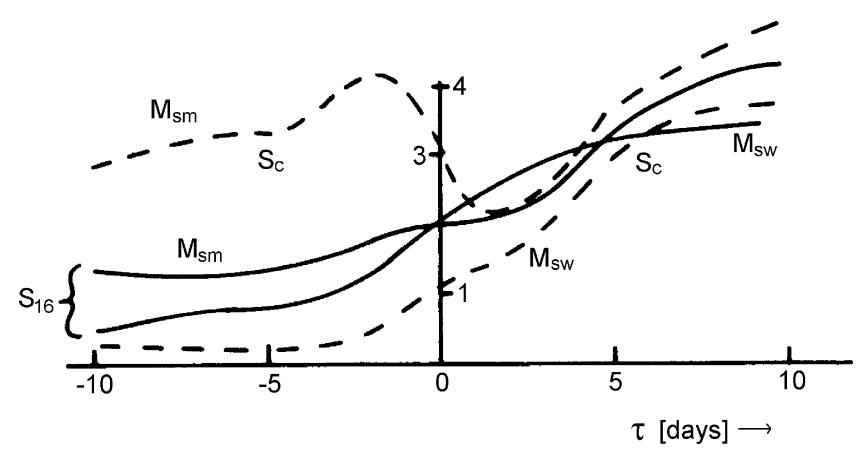

Fig. 3. Covariances $C\left(F, M_{\mathrm{sw}}, \mathrm{m} \mid \tau\right)$ of the global stratospheric angular momentum with the normalized flux $F$ through $\Sigma_{\mathrm{T}}$ in $10^{5}$ Hadley s both for $S_{16}$ (full) and $S_{\mathrm{c}}$ (dashed) as lower boundaries of the stratosphere. 
must be partly due to horizontal fluxes through the vertical sections of $S_{\mathrm{c}}$ at $|\varphi|=\left|27^{\circ}\right|$ which transport stratospheric angular momentum back into the tropical upper troposphere. Moreover, the stratosphere's share of the tropical atmosphere is larger for the choice $S=S_{\mathrm{c}}$. Altogether, Fig. 3 demonstrates in accord with Fig. 2 that the angular momentum exchange events in the tropics have a fairly long life time. An extension of Fig. 3 to $|\tau|=20$ days would not result in a pronounced reduction of the values. For example, $M_{\mathrm{sm}}=4.58 \times 10^{5}$ Hadley s for $\tau=20$ days.

These long time scales dominate also the evolution of the anomaly patterns of $\mu$. At $\tau=-10$ days there is a positive equatorial anomaly in the troposphere surrounded by negative anomalies (Fig. 4a). In particular, there are pronounced equatorial minima at the surface and in the lower stratosphere. Twenty days later (Fig. 4b), the positive anomaly extends into the lower equatorial stratosphere. The amplitudes of the positive anomalies are larger than at $\tau=-10$ days. Otherwise, little changes occur within these 20 days. The same is true with respect to the mass term. As can be seen from Fig. 5, it is essentially the mass term, which is responsible for the minimum of $\mu$ near the ground. Moreover, the mass term is positive and grows in the lower stratosphere. Altogether, there is an overall increase of positive angular momentum above $\Sigma_{\mathrm{T}}$ which is reflected in the global budgets in Fig. 3. The mass term contributes to this increase.

The covariance $C(F, p \mid \tau)$ of pressure $p$ follows from that of the mass term by assuming hydrostatic balance

$$
\frac{\partial}{\partial z} C(F, p \mid \tau)=-g C(F, \rho \mid \tau)
$$

and a vanishing pressure perturbation on top. This leads to negative surface pressures of $\sim-20 \mathrm{~Pa}$ in the tropics for $|\tau| \leq 10$ days. These negative pressures are found up to a height of $\sim 3 \mathrm{~km}$. The negative mass terms close to the ground in Fig. 5 imply an increase of the pressure perturbation with height, which is found indeed to extend up to the top with maximum values of $10 \mathrm{~Pa}$ in the tropical troposphere. Temperature covariances are positive throughout the tropical troposphere with a cool patch $(T \geq-0.2 \mathrm{~K})$ at and above the tropical tropopause. Since $C(P, p \mid \tau)>0$ there, these negative temperature perturbations reflect the positive density values found there (Fig. 5).

The related flux structures are displayed in Fig. 6, where, of course, upward motion is seen at the tropical tropopause at $\tau=0$ linked to an anticyclonic cell of $\psi$ in the northern hemisphere and a cyclonic one in the south. The strongest upward fluxes occur in the troposphere. They become weaker with increasing height above $z \sim 10 \mathrm{~km}$ but extend, of course, into the stratosphere. The $\psi$ - fluxes clearly contribute to the flux through $\Sigma_{\mathrm{T}}$. the low-level inflow towards the equator is, of course, related to the pressure minimum found there. The $\chi$-pattern at $\tau=0$ is centered at a positive anomaly near the equatorial tropopause but is clearly of global character with upward fluxes throughout the global troposphere (Fig. 6b). This upward component is particularly strong in the tropical belt. There is horizontal transport of AM out of the stratosphere through the vertical sections of $S_{\mathrm{c}}$. It is this transport which causes $M_{\mathrm{sm}}$ to decrease near $\tau=0$ (see Fig. 3). Moreover, the gradient of $\chi$ has a horizontal component directed outward from the equatorial domain in the lower troposphere. The flux patterns weaken quickly with increasing $\tau$.

The flux events are supported by torques centered in the tropics. Although, we cannot separate friction and mountain torques in Fig. 6, the presentation of the global torques in Fig. 7 suggests that both the mountain and the friction torque are important at $\tau=0$. The contribution by the mountain torque is negative for $\tau<-7$ days but becomes quickly large and positive. Nevertheless, the friction torque pumps more positive angular momentum into the atmosphere in the interval $|\tau| \leq 10$ days $\left(\sim 1.9 \times 10^{6}\right.$ Hadley s). This input is larger than the stratospheric gain of angular momentum. Most of it contributes to a growth of $M_{\mathrm{T}}$ (not shown). 

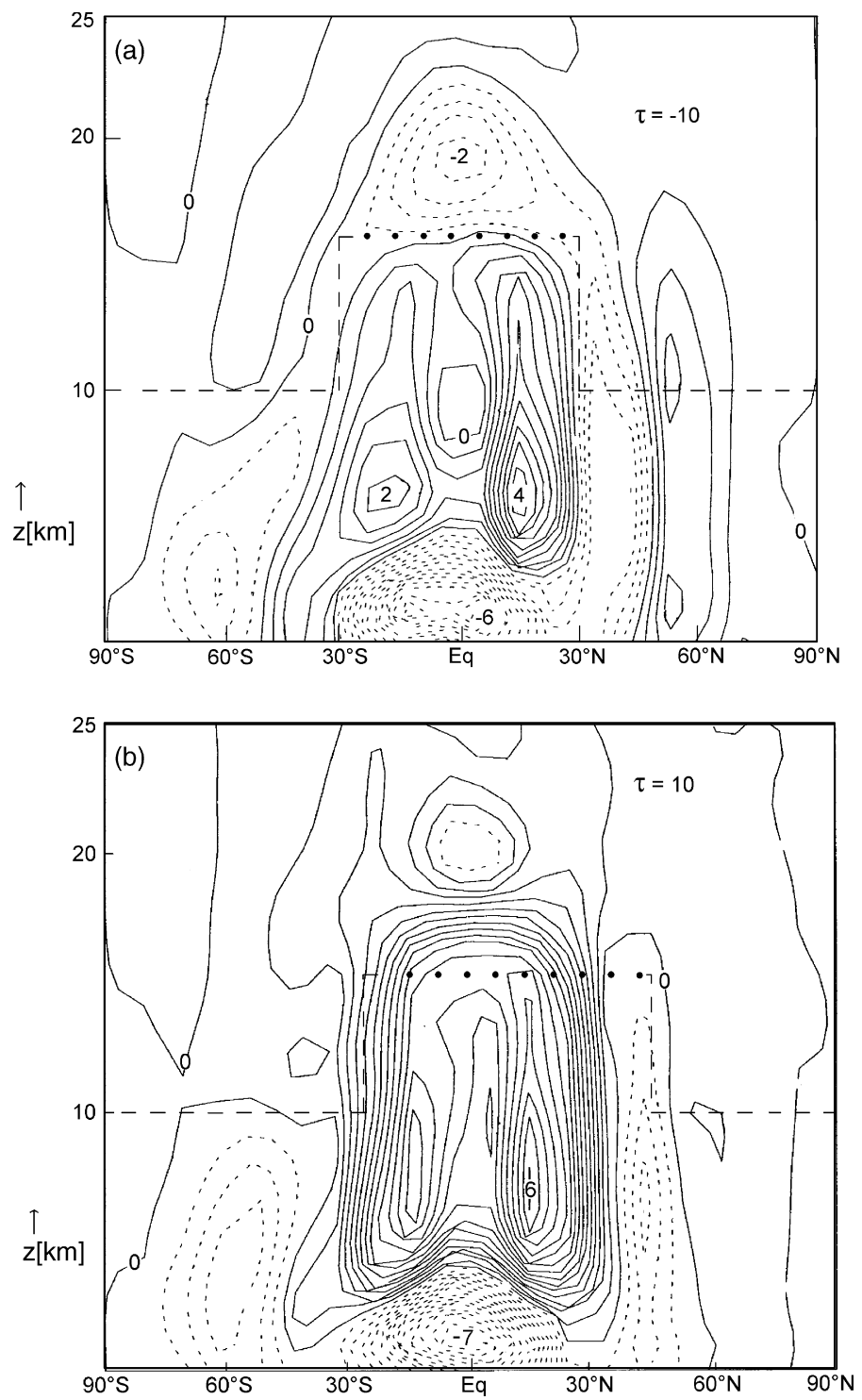

Fig. 4. Covariance $C(F, \mu \mid \tau)$ of the normalized flux through $\Sigma_{\mathrm{T}}$ (dots) with $\mu$ in $10^{4}$ Hadley s at (a) $\tau=-10$ days; (b) $\tau=10$ days. Contour interval $4 \times 10^{3}$ Hadley s. The dashed line gives the climatological tropopause $S_{\mathrm{c}}$.

Altogether, the flux events at $\Sigma_{\mathrm{T}}$ include phases of short-term intensification of the Hadley circulation with divergence of AM fluxes in the lower equatorial troposphere and convergence above with strong fluxes through the vertical parts of $S_{\mathrm{c}}$. Pressure and temperature fields are compatible with the notion of deep convective activity. The growth of anomalous positive angular momentum in the equatorial belt extends into the stratosphere during this process. The midlatitudes participate in this exchange process. There is also a weaker longterm component. Results change surprisingly little if $F$ is replaced by $F^{+}$or $F^{-}$. This means that positive flux events represent negative ones fairly well except for changes of signs. 


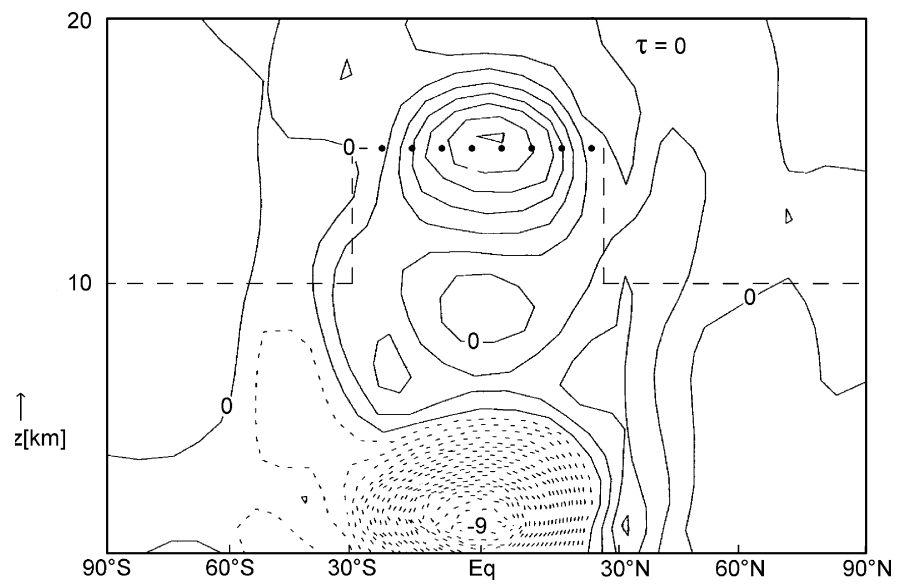

Fig. 5. Covariance $C\left(F, \mu_{\mathrm{m}} \mid \tau\right)$ of the normalized flux through $\Sigma_{\mathrm{T}}$ (dots) with the mass term $\mu_{\mathrm{m}}$ in $10^{4}$ Hadley s at $\tau=0$ days. Otherwise as in Fig. 4.

\subsection{Midlatitude events}

With $\Sigma_{\text {SH }}$ (Table 1; Fig. 9) we select a flux domain in the southern hemisphere covering the midlatitudes there. The autocorrelation of the flux through $\Sigma_{\mathrm{SH}}$ decays fairly quickly (Fig. 2) and one may attach a total lifetime of 8-10 days to flux events. The standard deviation of the flux is $\sigma_{\mathrm{F}}=40$ Hadley, that is, midlatitude fluxes tend to be much larger than equatorial ones.

The stratospheric wind term for $S_{\mathrm{c}}$ is quite small for $\tau<-3$ days and grows quickly till $\tau=1$ days, but does not vanish for $\tau=10$ days (Fig. 8). However, $M_{\mathrm{sw}} \sim 0$ at $\tau=20$ days (not shown). The mass term decreases slightly so that the total gain of the stratospheric angular momentum is quite small $\left(5 \times 10^{4}\right.$ Hadley s) despite the fact that $\sigma_{\mathrm{F}}$ is so large. Downward fluxes outside of $\Sigma_{\mathrm{SH}}$ must compensate much of the upward flux through $\Sigma_{\mathrm{SH}}$.

The global distribution of the angular momentum exhibits a columnar structure where anomalies of either sign extend from the surface up to heights of $\sim 15 \mathrm{~km}$. This indicates a strong coupling of troposphere and stratosphere. At $\tau=-5$ days (Fig. 9a), positive anomalies are found in the subtropics of the southern hemisphere and negative ones at midlatitudes. Five days later, a center of positive anomalies centered near the tropopause is sandwiched between an extended equatorial domain of negative covariances and the remnants of the negative anomaly in the south. Well defined columns are found at $\tau=5$ days (Fig. $9 \mathrm{~b}$ ). The gain of stratospheric angular momentum from $\tau=-5$ days till $\tau \sim 0$ days occurs close to the vertical part of the 'tropopause'.

The $\psi$-field at $\tau=0$ (Fig. 10a) is dominated by an anticyclonic cell which pumps angular momentum upward through the control surface with corresponding downward transports in the tropical troposphere. The $\chi$-pattern reveals that we consider here a situation of overall downward directed $\chi$-gradients (Fig. 10b). There is a minimum of $\chi$ in the equatorial tropopause transition zone. Note that the $\chi$-gradients in this extratropical case are smaller than in the tropical one (Fig. 6b) just as the changes of $M_{\mathrm{S}}$ are smaller. Negative torques act in the subtropics. This raises the question why $M_{\mathrm{S}}$ increases despite this overall downward flux. Obviously, the horizontal transports out of the tropical troposphere into the stratosphere at $\varphi=27^{\circ} \mathrm{S}$ are larger than the downward fluxes through the level parts of the 'tropopause'. In particular, the flux through $\Sigma_{\mathrm{SH}}$ related to $\chi$ is also negative. Note the similarity of Figs. $6 \mathrm{~b}$ and $10 \mathrm{~b}$ except for the sign. The 

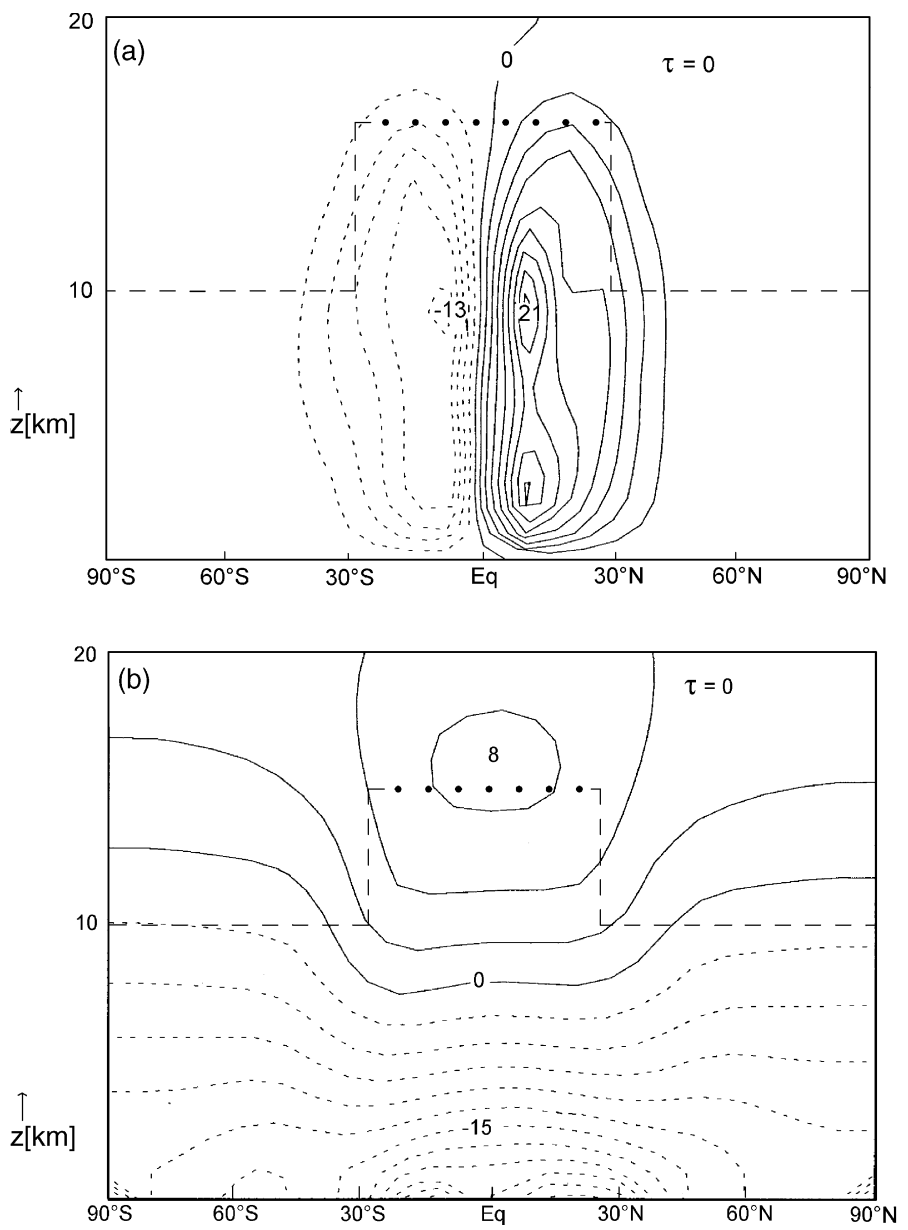

Fig. 6. Covariance fields (a) $\psi$ in Hadley (contour interval 2.5 Hadley) and (b) $\chi$ in 0.1 Hadley (contour interval 0.25 Hadley) at $\tau=0$ for fluxes through $\Sigma_{\mathrm{T}}$ (dots); $S_{\mathrm{c}}$ dashed.

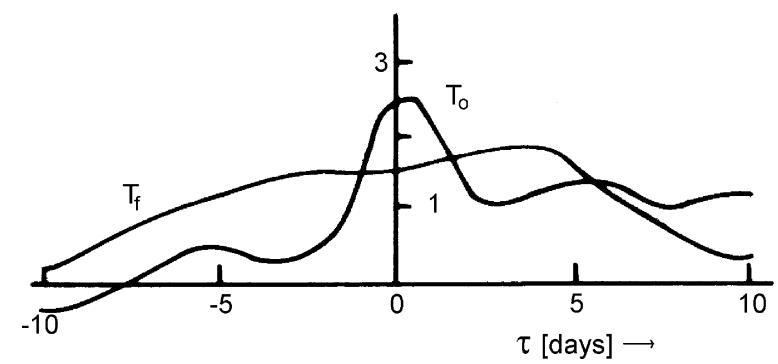

Fig. 7. Covariances of the flux through $\Sigma_{\mathrm{T}}$ with the global mountain torque $T_{\mathrm{o}}$ and the global friction torque $T_{\mathrm{f}}$ as a function lag in Hadley. 


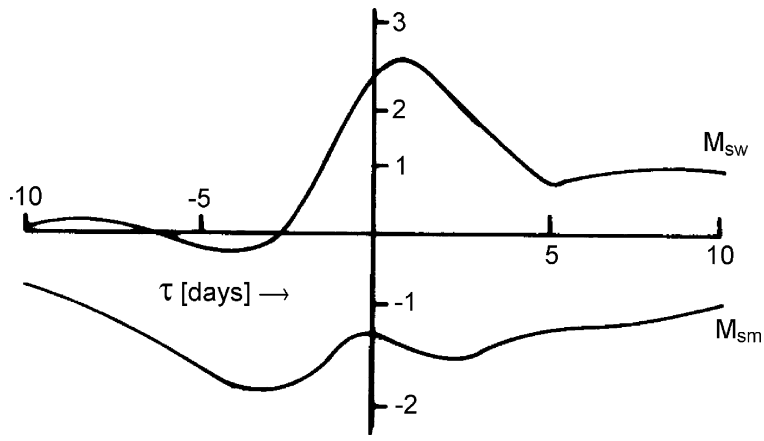

Fig. 8. Covariances $C\left(F, M_{\mathrm{sw}, \mathrm{m}} \mid \tau\right)$ of the normalized flux $\mathrm{F}$ through $\Sigma_{\mathrm{SH}}$ with the global stratospheric angular momentum in $10^{5}$ Hadley s for the stratosphere with lower boundary $S_{\mathrm{c}}$.
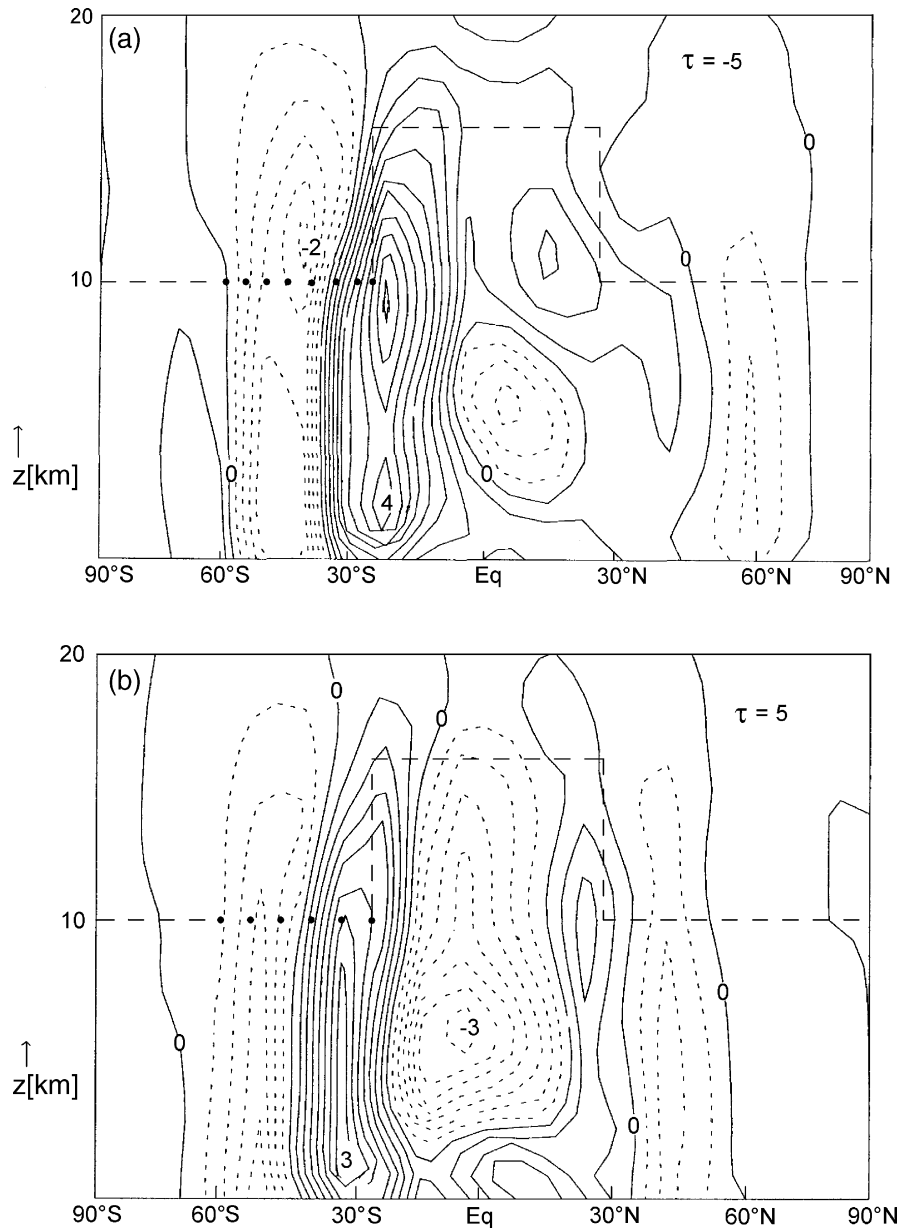

Fig. 9. Covariances $C(F, \mu \mid \tau)$ of the normalized flux $F$ through $\Sigma_{\mathrm{SH}}$ (dots) with $\mu$ in $10^{4}$ Hadley s at (a) $\tau=-5$ days and (b) $\tau=5$ days. Contour interval $14 \times 10^{3}$ Hadley s. Dashed: $S_{\mathrm{c}}$. 

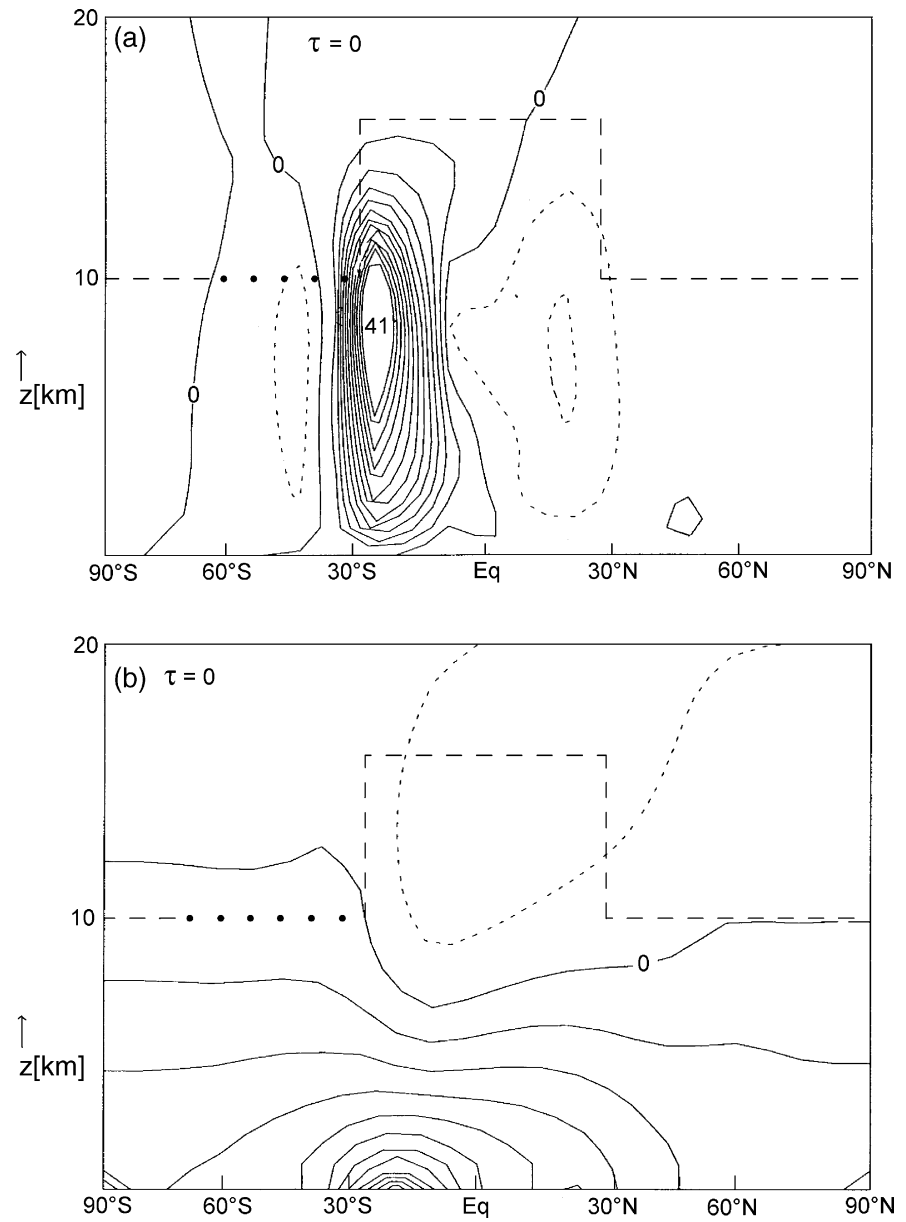

Fig. 10. Covariance fields (a) $\psi$ in Hadley (contour interval 2.5 Hadley) and (b) $\chi$ in 0.1 Hadley (contour interval 0.25 Hadley) at $\tau=0$ for fluxes through $\Sigma_{\mathrm{SH}}$ (dots); dashed: $S_{\mathrm{c}}$.

$\psi$-fluxes in Fig. 6a imply a negative flux through $\Sigma_{\mathrm{SH}}$. That explains the switch of signs at least partly.

The corresponding case in the northern hemisphere $\left(\Sigma=\Sigma_{\mathrm{NH}}\right)$ is so similar in most respects to that in the south that a specific discussion is not warranted. This case reveals, however, an even more pronounced link between the tropics and extratropics than the flux events at $\Sigma_{\mathrm{SH}}$. Fig. 11 shows the covariance of the flux through $\Sigma_{\mathrm{NH}}$ and $\mu$ at $\tau=10$ days. This pattern is almost symmetric with respect to the equator. The $\mu$-values in the northern column are somewhat larger than those in the southern one but the overall symmetry is striking. The message is clear: although one may formally define regional segments $\Sigma$ for flux events, it turns out that the related flux patterns are almost global and involve always the equatorial domain. The patterns in Fig. 9 are reminiscent of the structures found by Feldstein (2001) and others during strong synoptic wave events but the tight coupling of the fluxes at $\Sigma_{\mathrm{SH}}$ and $\Sigma_{\mathrm{NH}}$ with equatorial dynamics appears to be new. A switch to $F^{+}$has little impact in all cases discussed in this section. 


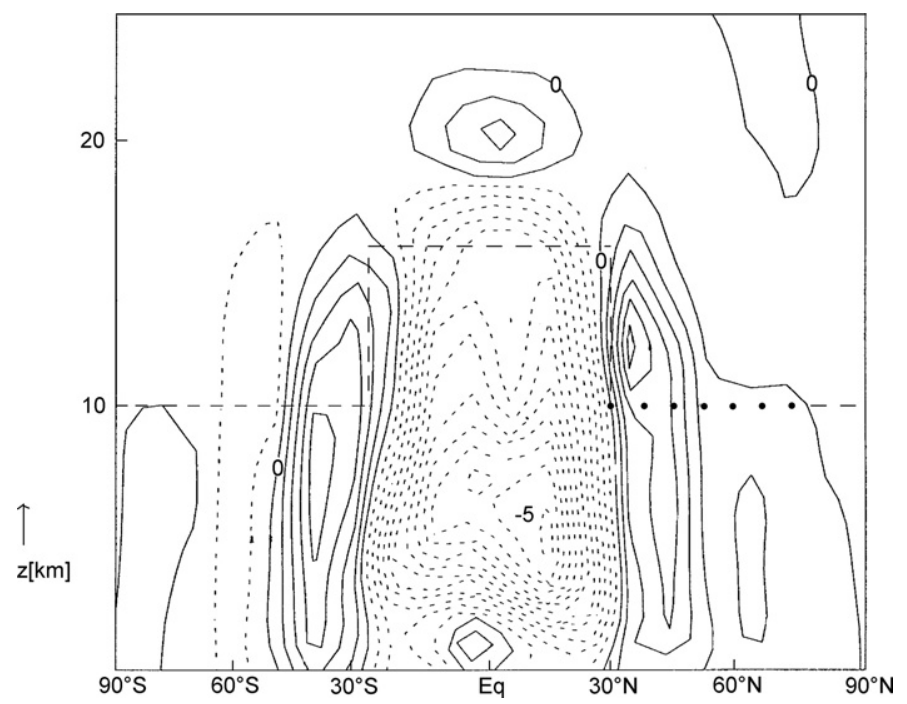

Fig. 11. Covariance $C(F, \mu \mid \tau)$ of the flux through $\Sigma_{\mathrm{NH}}$ (dots) with $\mu$ in $10^{4}$ Hadley s at $\tau=0$; contour interval $4 \times 10^{3}$ Hadley s; dashed: $S_{\mathrm{c}}$.

\subsection{Global events}

A new feature enters our analysis when $\Sigma$ coincides with the climatological tropopause $S_{\mathrm{c}}$ because horizontal fluxes through the vertical section contribute to the total flux in this case. The autocorrelation of the total flux decays quite rapidly with lag (not shown). This implies that the horizontal flux contributions are important. Otherwise the transports through $\Sigma_{\mathrm{T}}$ with their long timescales would presumably dominate. It is, however, difficult to draw conclusions with respect to $S_{\mathrm{c}}$ on the basis of what has been shown so far. Events with strong fluxes through a section of $S_{\mathrm{c}}$ need not be events of strong fluxes through the whole of $S_{\mathrm{c}}$. In particular, the standard deviation is relatively small with $\sigma_{F}=18$ Hadley. This reflects the fact, that the fluxes related to the $\psi$-field cancel in the global mean while they are quite important in all other cases.

The global wind term increases rapidly near $\tau=0$ and stays constant for $-10 \leq \tau \leq-3$ days and $2 \leq \tau \leq 10$ days (Fig. 12). The mass term decreases almost monotonously from $\tau=-10$ days till $\tau \sim 2$ days to recover the loss lateron. The bump of $M_{\mathrm{sw}}$ and the dip of $M_{\mathrm{sm}}$ near $\tau=2$ days suggest that 'Coriolis torques' are acting. There is a total increase of stratospheric angular momentum by $3.4 \times 10^{5}$ Hadley s. The flux through $S_{\text {c }}$ provides a total input of $6 \times 10^{5}$ Hadley s within the interval $-10 \leq \tau \leq 10$ days. Thus, the flux calculation predicts a stratospheric response twice as large as observed. The angular momentum patterns for $\tau<0$ exhibit a buildup of deep columns of positive $\mu$ on the poleward side of the steps and of a deep negative anomaly centered at the equator (Fig. 13). The $\mu$-distribution at $\tau=10$ days is fairly similar to that at $\tau=0$. This pattern is, of course, strongly reminiscent of Fig. 11. The stratospheric gain of angular momentum occurs close to the step. Obviously, angular momentum is flowing poleward out of the equatorial zone in this process. Part of this momentum is counted as stratospheric.

The anomalies of the streamfunction are centered underneath the steps (Fig. 14a). There are large imports at the 'tropopause breaks' which are balanced by exports through the level sections of $S_{c}$. The $\chi$-distribution at $\tau=0$ (Fig. 14b) illustrates the outflow of angular momentum from the 


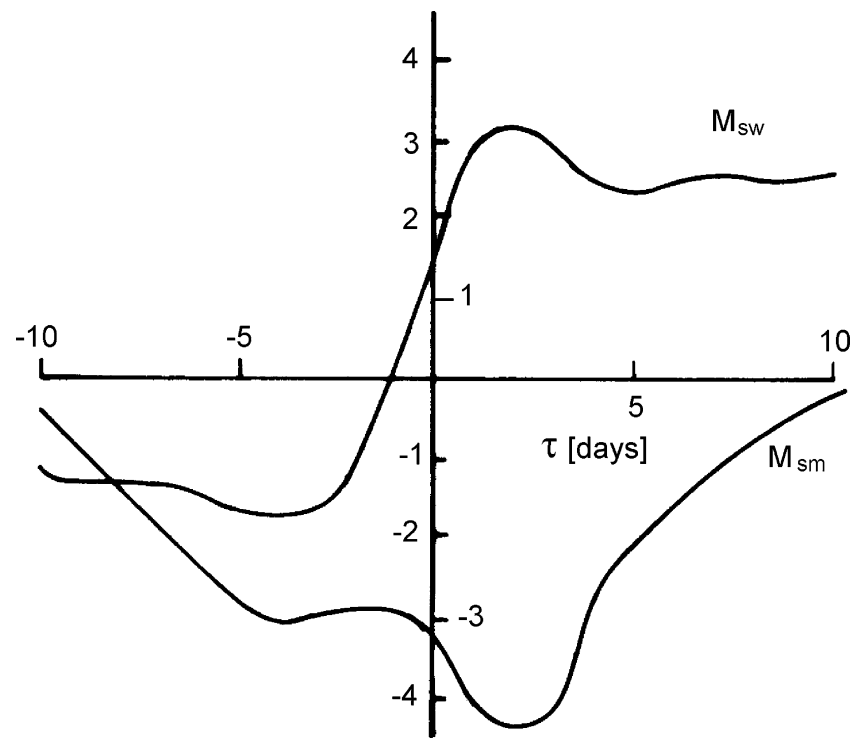

Fig. 12. Covariances $C\left(F, M_{\mathrm{sw}}, \mathrm{m} \mid \tau\right)$ of the normalized flux $F$ through $S_{\mathrm{c}}$ with the related global angular momentum component in $10^{5}$ Hadley s.

equatorial troposphere. Note the narrow ridges of $\chi$ near the steps which represent the horizontal flow through the step which converges, however, with upward and equatorward fluxes. These sharp ridges are not well resolved by the data grid. Imbalances in the budget can be assessed locally by inserting the data in (2.15) and calculating the residual where the time derivatives are replaced by centered differences. The residual, when multiplied by the time step can be compared to $C(F, \mu \mid \tau)$. It turns out that the residual is small $(\leq 10 \%)$ for, say, $\tau= \pm 5$ days but is quite large throughout the atmosphere near $|\varphi|= \pm 27^{\circ}$, where it locally exceeds $C(F, \mu \mid \tau)$.

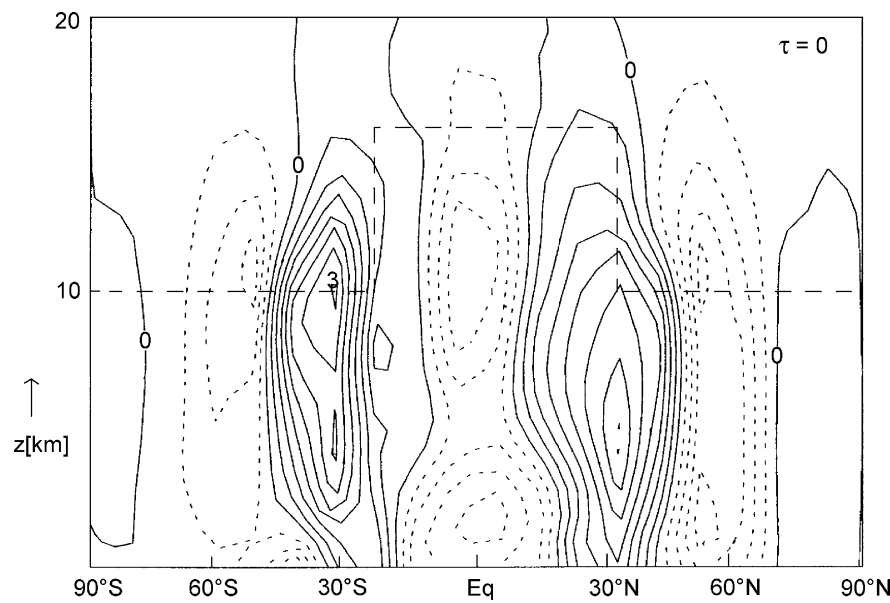

Fig. 13. Covariance $C(F, \mu \mid \tau)$ of the flux through $S_{\mathrm{c}}$ (dashed) with $\mu$ in $10^{4}$ Hadley s at $\tau=0$; contour interval $4 \times 10^{3}$ Hadley sec. 

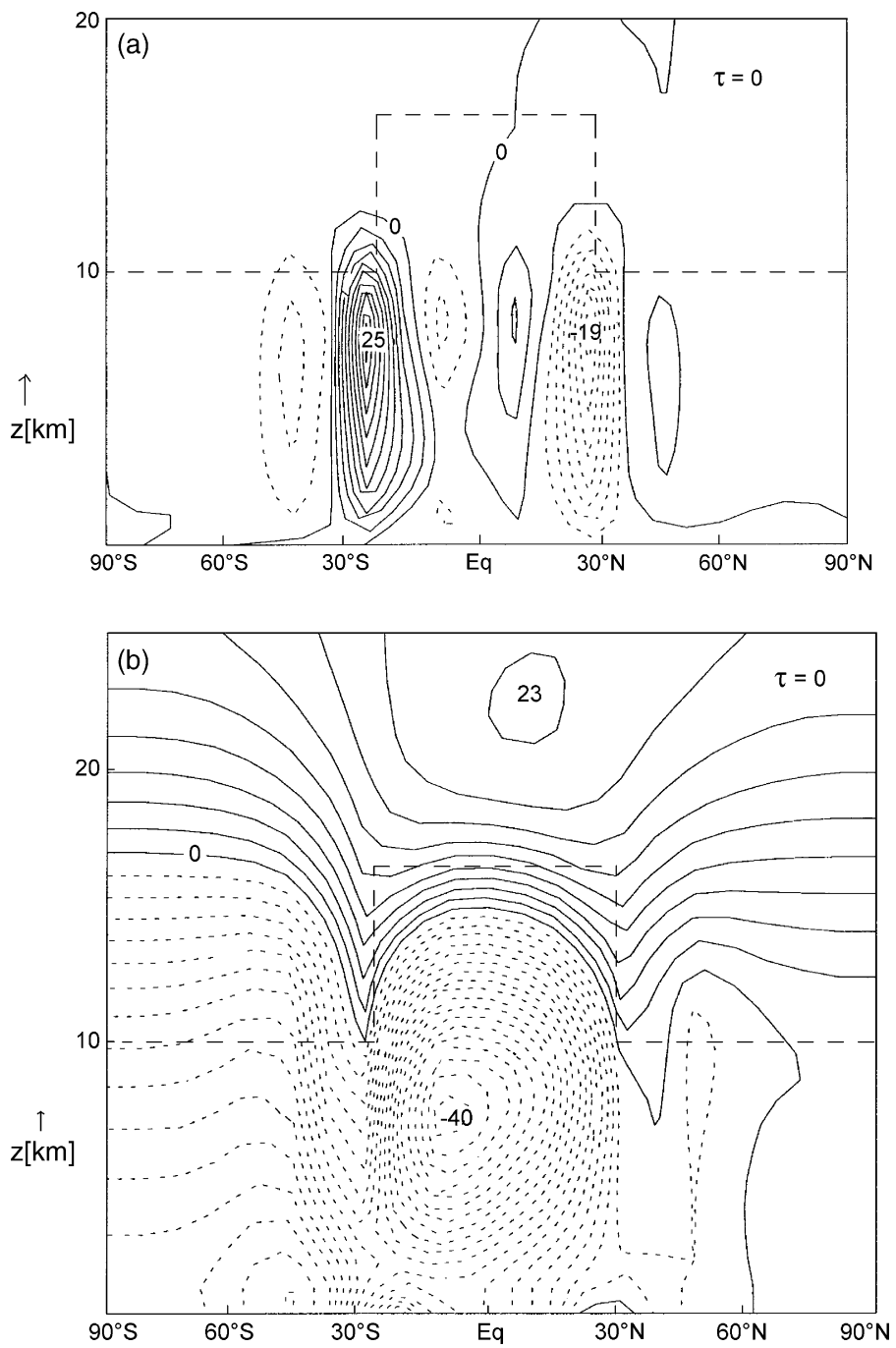

Fig. 14. Covariance fields (a) $\psi$ in Hadley (contour interval 2.5 Hadley and (b) $\chi$ in 0.01 Hadley (contour interval 0.0025 Hadley) at $\tau=0$ for fluxes through $S_{\mathrm{c}}$ (dashed).

That means that (2.15) cannot be used to calculate $\chi$. That has not been done as stated above. Instead, $\chi$ is calculated from the observed tendencies. Thus, the somewhat extreme $\chi$-pattern in Fig. 14b is trustworthy provided we believe that the angular momentum data are essentially correct. The zonal mean temperature deviations in this global case are fairly small. The ascending branches of the extratropical fluxes in Fig. 14a are cool with minima of $C(F, T \mid 0) \sim-015 \mathrm{~K}$. At the equator temperatures are again positive close to the ground and negative above. The results for positive events where $F$ is replaced by $F^{+}$differ little from what has been shown here.

With $\Sigma=\mathrm{S}_{16}$ we recover essentially the situation found in the case with $\Sigma_{\mathrm{T}}$ but the standard deviation of the flux is quite small ( $\sigma_{F}=3$ Hadley). 


\section{Conclusion and discussion}

It is appropriate to list first some shortcomings of our approach before trying to summarize the results. First of all, it must be stated that cause and effect can hardly be disentangled because we concentrate on one dynamical variable. We are not really able to go beyond an advanced description of the phenomena although angular momentum with its conservation properties is certainly a suitable variable for such an approach. Second, the analysis is restricted to 'tropopauses' with specified, constant positions. A day-by-day calculation of fluxes through a realistic twodimensional tropopause is to be preferred to this rather schematic approach. The computational effort increases, however, drastically in this case. Moreover, it is not obvious if the data quality is sufficient to warrant this effort. The statistical budget equation (2.15) is not satisfied by the data with a high degree of accuracy. Correspondingly, adjustment procedures are common in angular momentum budgets where the vertical fluxes are adapted (e.g., Egger and Hoinka, 2005b). Such procedures have been avoided here.

Extratropical STE is associated with synoptic-scale and mesoscale processes and is, therefore, highly intermitted and localized (e.g., Stohl et al., 2003). Such processes are, of course, represented to some extent also in the zonal means on which our analysis is based. However, our selection of fairly wide segments $\Sigma$ smears localized features out. It is conceivable that our approach misses some of the STE events linked to cut-offs and PV-streamers in midlatitudes (e.g., Stohl et al., 2003; Sprenger et al., 2005). The deep columnar structures of the $\mu$-field as displayed in Figs. 9 and 11 are not typical of these STE events. It is our statistical technique, which allows us to investigate these STE events of rather large scale.

There is an ongoing debate with respect to the impact of the stratosphere on the troposphere and eventual troposphere-stratosphere-troposphere coupling (see Reichler et al., 2005, for a most recent contribution). Our contribution to this debate is straightforward. Given a flux event, we may look at covariances for $\tau \sim-\tau_{\mathrm{e}}$ where $\tau_{\mathrm{e}}$ is the decay time of the autocorrelation of the flux. If anomalies at that lag are much more concentrated, say, in the stratosphere than at $\tau=0$, this is an indication that the flux event originates in the stratosphere. Such signals are not seen in our analysis. All flux events develop jointly and involve both the stratosphere and the troposphere. Moreover, they tend to be of global character.

The tropical flux events involve mass fluxes throughout the tropical tropopause layer and are tied to a short-term intensification (weakening) of the Hadley circulation. The effect on the stratospheric angular momentum is, however, global. There is also a slow longterm component which is difficult to identify. One may think of the Madden-Julian-Oscillation (MJO), but Fig. 4 does not show the corresponding features of an oscillation nor fits Fig. 4 the known changes of the angular momentum during an MJO-cycle (Weickmann et al., 1997).

All events involve strong fluxes at the 'tropopause breaks' which are not balanced by corresponding return fluxes at other parts of the tropopause. The fluxes through the breaks contribute to an increase of stratospheric AM in the midlatitude cases while angular momentum is transported out of the stratosphere elsewhere. Conversely, stratospheric AM is lost through the breaks in the tropical case. Data accuracy is barely sufficient in the global case. That notwithstanding, our analysis demonstrates that substantial fluxes of angular momentum must occur at the tropopause breaks. There is a close link of the midlatitude events to the equatorial domain.

All this suggests that we have been looking at fairly similar modes of angular momentum exchange throughout this paper. They all involve centers of flux convergence or divergence in the lower equatorial stratosphere with corresponding global $\chi$-fluxes toward or away from these centers. Moreover, there is always a deep equatorial anomaly of angular momentum extending into 
the lower stratosphere sandwiched between columns of opposite sign in the north and south. The similarity of the $\psi$-patterns is not so pronounced because these capture mainly the vertical AM fluxes underneath the $\Sigma$-surface chosen. On the other hand, positive (negative) torques support the flux convergence (divergence) in the lower equatorial stratosphere. In other words, we may have found a global mode of STE.

It is tempting to explain the AM flow patterns presented in this paper in terms of quasilinear arguments, where covariances consist of a dominant 'linear' and a small 'turbulent' part. For example, the covariance of $C\left(F, \tilde{V}_{\mathrm{w}} \mid \tau\right)$ of the flux $F$ with the horizontal wind term flux can be separated according to the standard splitting

$$
V_{w}^{*}=\hat{\rho}\left(\left(\hat{u} \bar{v}^{*}+\hat{v} \bar{u}^{*}+\left(\hat{u^{\prime} v^{\prime}}\right)^{*}\right) \operatorname{acos} \varphi\right.
$$

where we disregard variations of density and where $(*)$ stands for a deviation from the time mean. The first two terms on the right of (4.1) are the quasilinear ones. It would be relatively easy to interpret them. The data allow us to perform the separation (4.1). It is found for $\Sigma_{\mathrm{T}}$, for example, that the first term on the right is much more important than the second one but the major part of the fluxes near the tropopause breaks (see also Fig. 6) at $\tau=0$ is due to the turbulent term in (4.1). This finding excludes quasilinear explanations of our results. A theory of the AM exchanges depicted here has to include contributions by large-scale turbulence. That is not surprising because all events discussed here involve strong fluxes through the 'tropopause breaks', which are certainly linked to large-scale threedimensional perturbations. It is, however, surprising that the results for positive flux events are so similar to those obtained for negative ones.

\section{Acknowledgments}

We are grateful to the anonymous referees for their helpful and constructive comments.

\section{References}

Baldwin, M., et al., 2001. The quasibiennial oscillation. Rev. Geophys. 39 (2), 179-229.

Egger, J., Hoinka, K.-P., 2002. Covariance analysis of the global atmospheric angular momentum budget. Mon. Wea. Rev. 130, 1063-1070.

Egger, J., Hoinka, K.-P., 2005a. The annual cycle of the axial angular momentum of the atmosphere. J. Clim. 18, 757-811.

Egger, J., Hoinka, K.-P., 2005b. Torques and the related meridional and vertical fluxes of axial angular momentum. Mon. Wea. Rev. 133, 621-633.

Feldstein, S., 2001. Friction torque dynamics associated with intraseasonal length-of-day variability. J. Atmos. Sci. 58, 2942-2953.

Fueglistaler, S., Wernli, H., Peter, T., 2004. Tropical troposphere-to-stratosphere transport inferred from trajectory calculations. J. Geophys. Res. 109, doi:10.1029/2003JD4069.

Gibson, R., Kållberg, P., Uppala, S., Hernandez, A., Nomura, A., Serrano, E., 1997. ERA description. ECMWF Reanalysis Project Rep. Ser. 1, 86 (Available from ECMWF, Shinfield Park Reading, RGZGAS, UK).

Haynes, P., 2005. Stratospheric dynamics. Ann. Rev. Fluid Mech. 37, 263-293.

Haynes, P., Marks, C., McIntyre, M., Shepherd, T., Shine, K., 1991. On the 'downward control' of extratropical diabatic circulations by eddy-induced mean zonal forces. J. Atmos. Sci. 48, 651-678.

Highwood, E., Hoskins, B., 1998. The tropical tropopause. Quart. J. Roy. Met. Soc. 124, 1579-1604.

Holton, J., et al., 1995. Stratosphere-troposphere exchange. Rev. Geophys. 33 (3), 403-439.

Hoskins, B., 1991. Towards a PV- $\theta$ view of the general circulation. Tellus 43 AB, 27-35.

James, P., Stohl, A., Forster, C., Eckhart, S., Seibert, P., Frank, A., 2003. A 15-year climatology of stratosphere-troposphere exchange with a Lagrangian particle dispersion model. 2. Mean climate and seasonal variability. J. Geophys. Res. 108 (D12), 8522, doi:10.1029/2002D002639.

Kim, H., Lee, S., 2004. The wave-zonal mean flow interaction in the southern hemisphere. J. Atmos. Sci. 61, $1055-1067$. 
Lamarque, J., Hess, P., 1994. Cross-tropopause mass exchange and potential vorticity budget in a simulated tropopause folding. J. Atmos. Sci. 51, 2246-2269.

Peixoto, J., Oort, A., 1992. Physics of Climate. Am. Inst. Phys., 520.

Price, J., Vaughan, G., 1993. On the potential for stratosphere-tropopause exchange in cut-off-low systems. Quart. J. Roy. Met. Soc. 119, 343-365.

Reichler, Th., Kushner, P., Polvani, L., 2005. The coupled stratosphere-troposphere response to impulsive forcing from the troposphere. J. Atmos. Sci. 62, 3337-3352.

Rosenlof, K., Holton, J., 1993. Estimates of the stratospheric residual circulation using the downward control principle. J. Geophys. Res. 98 (D6), 10465-10479.

Shepherd, Th., Shaw, T., 2004. The angular momentum constraint on climate sensitivity and downward influence on the Middle Atmosphere. J. Atmos. Sci. 61, 2899-2908.

Sprenger, M., Wernli, H., Bourqui, M., 2005. Cross-tropopause mass exchange and its relation to potential vorticity streams and cut-offs near the extratropical tropopause. J. Atmos. Sci., to appear.

Stohl, A., et al., 2003. Stratosphere-troposphere exchange: A review, and what we learned from STACCATO. J. Geophys. Res. 108 (D12), 8516, doi:10.1029/2002JD002490.

Traub, M., Lelieveld, J., 2003. Cross-tropopause transport over the eastern Mediterranean. J. Geophys. Res. 108 (D2), art. No. 4712, doi:10.1029/2002JD 002587.

Weickmann, K., Kiladis, G., Sardeshmukh, P., 1997. The dynamics of intraseasonal atmospheric angular momentum oscillations. J. Atmos. Sci. 54, 1445-1461.

Wernli, H., Bourqui, M., 2002. A Lagrangian "1-year climatology" of (deep) cross-tropopause exchange in the extratropical Northern Hemisphere. J. Geophys. Res. 107 (D2), 4041, doi:10.1029/2001JD00812.

Wirth, V., Egger, J., 1999. Diagnosing extratropical synoptic-scale stratosphere-troposphere exchange: a case study. Quart. J. Roy. Met. Soc. 125, 635-655. 